\title{
Long-Tube Method for Field Determination of Sound-Absorption Coefficients
}

\author{
Earle Jones, Seymour Edelman, and Albert London*
}

\begin{abstract}
A method has been developed that makes it possible o measure the sound-absorption coefficient of acoustic materials that have been installed. The method uses a portable version of the familiar impedance tube, in which measurements on the sianding sound-wave pattern are used to obtain the sound-absorption coefficient for normally incident sound. The sound-absorption coefficient for randomly mcident sound may be determmed from the tube measurements by reference to an extensive table given in the paper. The tube is coupled to the acoustic material without cefacing its surface so that the test is nondestructive. It is usetul for acceptance testing; the determination of the effects of aging, staining, and redecoration of acoustic materials; and for quality-control purposes in the manufacture of acoustic materials. The results of laboratory measurements on a large number of acoustic tiles and plasters were compared with the results obtained with the standard reverberationroom techniques. Field measurements were made on acoustic plasters. Large variations in the absorption coefficient were observed and ascribed to faulty application and painting procedures. Measurements were made at 512 cycles per second only.
\end{abstract}

\section{Introduction}

The question of how well installations of acoustic materials comply with specifications written on the basis of laboratory tests is one of considerable importance. Laboratory samples of acoustic plasters are constructed with care under controlled conditions and usually yield repeatable results, even when tested by different laboratories. How ever, when the materials are installed the sound-absorption coefficients depend to a large degree on such factors as the care devoted to the mixing of the ingredients, the skill of the plasterer, and the conditions under which the plaster is cured. Any one of these factors may materially affect the sound-absorbing properties of the installation.

The problem of maintenance of acoustic-material installations is also of great economic importance to large-scale users of acoustic materials, like the Federal Government. The General Services Administration and the Veteran's Administration have many acres of acoustic material to care for. When this material becomes soiled or otherwise unsightly with the passage of time, the choice of a poor method to restore its appearance may result in the loss of its sound absorption. In experimenting with various methods of redecoration, it is advantageous to make field measurements of the sound absorption of a small portion of the material before and after using a particular method of redecoration because it is difficult to reproduce aging and staining conditions in the laboratory.

The "long-tube method" described in this paper makes it possible to measure the sound-absorption coefficient of acoustic materials that have been installed without defacing them. It consists of setting up a standing sound-wave pattern within a tube, one end of which is closed by the acoustic material being: tested, and, from measurements of the maximum and minimum pressures in the standing wave pattein, computing the sound-absorption coefficient for nor- mal incidence. A calculation based on semiempirical considerations is then used to determine, from the normal incidence absorp ion coefficient, the random incidence absorption coefficient that corresponds to the value that would be determined by a reverberation-room test. An explanation of this calculation is given in the paper by London. ${ }^{1}$

\section{Equipment}

In designing the tube, $512 \mathrm{c} / \mathrm{s}$ was chosen as the operating frequency because this frequency is used in reverberation testing, and it is near the mean of the frequency range used in sound-absorption tests.

It was desired to have the diameter of the tube as large as practicable in order to make measurements on as large a sample as possible and in order to reduce the effects of the attenuation due to the walls of the tube. The maximum diameter allowable was taken as just less than the cutoff diameter for the first transverse mode. It was desired to make the tube long enough so that at least two minima and one maximum could be found but not too long for easy handling. In view of these considerations, and limited by available material, it was decided that the tube should be made of $1 / 8$-in. brass tubing, $93 / 4$ in. in diameter, and about 30 -in. long. The sound source is a 6 -in. permanent-magnet speaker mounted on a 1/8-in. brass plate covering the back of the tube. Several types of microphones were tried. The most successful was a hearing-aid-type crystal microphone. This is mounted on the center bar of a metal $\mathrm{H}$-shaped member. The two parallel bars of the $H$-member are parallel to the axis of the tube and bear brass wheels that roll on tracks along the tube. Springs press the wheels against the tracks with sufficient pressure to hold the microphone in position if the tube is rotated from horizontal to vertical. The microphone is moved along the tube by means of a steel pipe fastened to the $\mathrm{H}$-member and passing through a

Determination of reverberant sound absorption coefficients from acoustic impedance measurements, J. Acous, Soc. Am. 22, 263 (1950). 
close-fitting bearing in the back cover of the tube. A fine adjustment of the position can be made by fastening the steel pipe by means of a setscrew in the back bearing and moving the $\mathrm{H}$-member by a steel rod threaded through the pipe.

The tube is pivoted so that it can be used at any angle between vertical and horizontal. The pivot supports are on a jackscrew so that the tube can be moved vertically about $1 \mathrm{ft}$. The entire mount is on casters for easy movement from place to place.

A bakelite housing cemented over the magnetic pot of the loudspeaker shields the back of the tube from extraneous sound. After much experimentation, it was found that a caulking-compound gasket would bond the tube to the material being tested without too much leakage of sound and without damaging the material.

Figure 1 is a circuit diagram of the setup. The signal from an oscillator drives the loud-speaker. The microphone signal is amplified, filtered to remove the effects of extraneous sound and harmonics, and read on a sensitive vacuum-tube volimeter having an accurate decibel scale. Measurements on successive minima in the tube indicate that damping at the tube wall is negligible because of the large diameter.

\section{Theory}

The expression for the normal incidence absorption coefficient in terms of the maximum and minimum pressures in the tube is ${ }^{2}$

$$
\alpha_{0}=1-\left[\frac{P_{\max }-P_{\min }}{P_{\max }+P_{\min }}\right]^{2}=\frac{4 P_{\max } P_{\min }}{\left[P_{\max }+P_{\min }\right]^{2}} .
$$

This expression can be written in terms of $r$, the ratio between $P_{\max }$ and $P_{\min }$, as

$$
\alpha_{0}=\frac{4 r}{[1+r]^{2}} .
$$

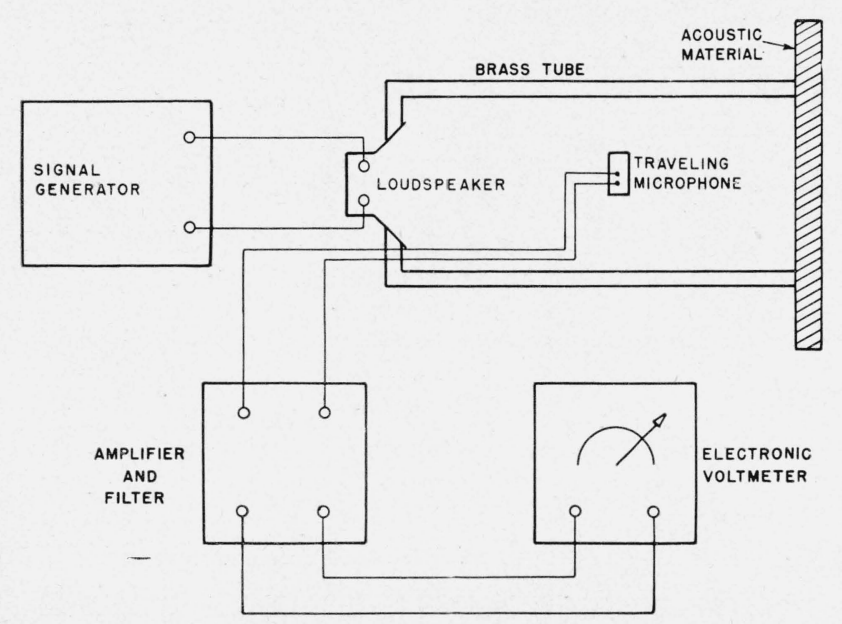

Figure 1. Block diagram of rube and associated equipment as used in field measurements of sound-absorption coefficient

${ }^{2}$ H. O. Taylor, Phys. Rev. [2] 2, 270 (1913).
In terms of $\Delta$, the difference in db between the maximum and minimum pressures, the expression is

$$
\alpha_{0}=\operatorname{sech}^{2}(A), \quad A=\frac{\Delta}{40 M}, \quad M=\log _{10} e .
$$

The relationship between $\alpha_{0}$ and $\alpha_{e}^{*}$, the absorption coefficient for random incidence, is given by London as

$$
\begin{aligned}
\alpha_{e}^{*}=4\left[\frac{1-\left(1-\alpha_{0}\right)^{1 / 2}}{1+\left(1-\alpha_{0}\right)^{1 / 2}}\right][\ln 2-1 / 2- & \\
& \left.\ln \left\{1-\left(1-\alpha_{0}\right)^{1 / 2}\right\}-\frac{\left(1-\alpha_{0}\right)^{1 / 2}}{2}\right]^{2} .
\end{aligned}
$$

In terms of $\Delta, \alpha_{e}^{*}$ can be written as

$$
\begin{aligned}
\alpha_{e}^{*}=4\left[\frac{1-\tanh A}{1+\tanh A}\right] & {[0.1931-} \\
& \left.\ln \{1-\tanh A\}-\frac{\tanh A}{2}\right] .
\end{aligned}
$$

From this relationship, table 1 was prepared

\begin{tabular}{|c|c|c|c|c|c|c|c|c|c|c|}
\hline $\mathrm{db}$ & 0 & 1 & 2 & 3 & 4 & 5 & 6 & 7 & 8 & 9 \\
\hline $\begin{array}{r}0 \\
10 \\
20 \\
30 \\
40 \\
50\end{array}$ & $\begin{array}{r}0.77 \\
.84 \\
.60 \\
.32 \\
.14 \\
.06\end{array}$ & $\begin{array}{r}0.80 \\
.83 \\
.56 \\
.30 \\
.13 \\
.05\end{array}$ & $\begin{array}{r}0.82 \\
.81 \\
.53 \\
.27 \\
.12 \\
.05\end{array}$ & $\begin{array}{r}0.84 \\
.79 \\
.50 \\
.25 \\
.11 \\
.05\end{array}$ & $\begin{array}{r}0.85 \\
.77 \\
.48 \\
.24 \\
.10 \\
.04\end{array}$ & $\begin{array}{r}0.86 \\
.74 \\
.45 \\
.22 \\
.09 \\
.04\end{array}$ & $\begin{array}{r}0.86 \\
.71 \\
.42 \\
.20 \\
.09 \\
.03\end{array}$ & $\begin{array}{r}0.86 \\
.69 \\
.39 \\
.19 \\
.08 \\
.03\end{array}$ & $\begin{array}{r}0.86 \\
.66 \\
.37 \\
.17 \\
.07 \\
.03\end{array}$ & $\begin{array}{r}0.85 \\
.63 \\
.34 \\
.16 \\
.07 \\
.03\end{array}$ \\
\hline
\end{tabular}
giving the value of $\alpha_{e}^{*}$, the reverberant soundabsorption coefficient, for values of $\Delta \mathrm{db}$ over the range of $\Delta \mathrm{db}$ usually found in testing acoustic materials.

TABLE 1. Absorption coefficient for reverberant sound, $\alpha_{e}{ }^{*}$, in terms of ratio of $P_{\max }$ to $P_{\min }, \Delta d b$

Example: Suppose observed difference in sound level, $\Delta d b$, between $P_{\max }$ and $P_{m i n}$ is $14 \mathrm{db}$. Table gives $\alpha_{e}{ }^{*}$ as 0.77 .

\section{Laboratory Check of Method}

Simulated field measurements were made on samples of some 50 acoustic materials previously tested in the Bureau's reverberation chamber. Three different specimens of each material were tested, and each specimen was tested three different times. The average difference between the results of the tube measurement and the reverberationroom results was found to be -0.07 . It was assumed that the tube method gave results too low by this amount, and +0.07 was applied as a correction to each coefficient measured by the tube. After this correction had been applied, the deviations of the tube measurements from the reverberation-chamber measurements ranged from +0.18 to -0.21 . The average of the absolute values of these deviations was 0.05 , and 70 percent of all of the deviations fell between +0.05 and -0.05 . The dispersion pattern of the deviations of the tube-derived $\alpha_{e}^{*}$ coefficient from $\alpha_{\mathrm{rev}}$ for these materials is presented in figure 2 . 
The samples used for the simulated field measurements included many types of prefabricated acoustic materials. No correlation could be found between the amount of the deviation and the type of surface of the acoustic tile or between the amount of the deviation and the thickness of the tile. If the samples used for the simulated field tests are a fair representation of all acoustic materials, it appears that the coefficient found by means of the tube method will be within \pm 0.05 of the coefficient found in the reverberation chamber about 70 percent of the time and within \pm 0.10 about 80 percent of the time.

As new materials are tested in the reverberation chamber, simulated field tests are made on the same samples so that the correction to be applied in the field is being revised and kept up to date.

\section{Field Procedure}

The field procedure used in determining the soundabsorption coefficient by means of the long-tube method is made clear in figure 3 , which shows the equipment in use, and also in the following description of a test made on a ceiling. When mounted on the casters, the top of the tube in the vertical position can be set at any height from about $5 \mathrm{ft} .2 \mathrm{in}$. to $5 \mathrm{ft} .10 \mathrm{in}$. It is therefore necessary to use a scaffold to raise the tube high enough to enable the top of the tube to reach the ceiling. The tube is mounted on the scaffold and the jackscrew run up until the top of the tube just touches the ceiling, care being taken to see that the end of the tube touches the ceiling evenly. The caulking-compound gasket is then pressed against the ceiling to make a firm seal, as shown in figure 3 . The oscillator is then tuned to $512 \mathrm{c} / \mathrm{s}$ by comparison with a tuning fork. The microphone is moved along the tube by the steel pipe until the reading on the vacuum-tube voltmeter indicates that it is in the neighborhood of a point of minimum pressure. The pipe is then clamped, and the microphone is moved by the threaded rod until the exact pressure minimum is found. The reading

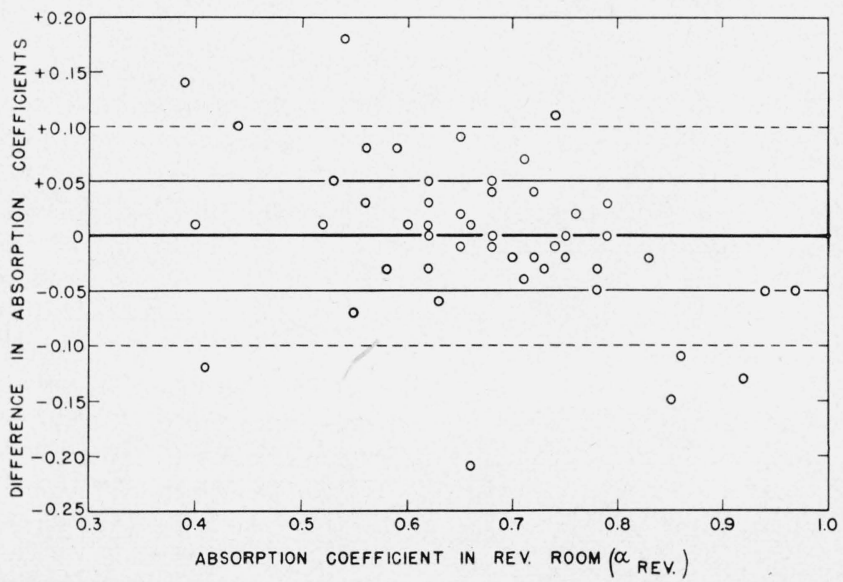

Figure 2. Plot of difference in sound-absorption coefficients measured by the long-tube method from the sound-absorption coefficients measured by the reverberation-room method for fifty different materials at $512 \mathrm{c} / \mathrm{s}$. of the voltmeter in decibels is recorded. The microphone is then moved in the same way to a point of pressure maximum, and the reading of the voltmeter is recorded. The difference in the two voltmeter readings is $\Delta \mathrm{db}$. The absorption coefficient corresponding to $\Delta \mathrm{db}$ is then found from the table and is corrected by adding 0.07 . As described in section 4 , the addition of 0.07 to the coefficients found by the tube method makes them agree, on the average, with values obtained in the reverberation room. This procedure is repeated at a large number of points over the ceiling, and the average of the values found is taken as the absorption coefficient of the material at $512 \mathrm{c} / \mathrm{s}$.

In a series of measurements made on acoustic plaster, it was found that the absorption coefficient varies from point to point by considerable amounts, and that the difference in absorption coefficient is closely correlated with differences in the appearance of the material. On acoustic plaster, it is therefore important to make measurements on as many different locations as practicable, and especially to sample as many points having different surface texture as practicable. Acoustic tile appears to be more uniform from one sample to another, and it may not be necessary to sample as many points on a tile sample as on a plaster sample.

If the tube is to be used for acceptance testing on a new installation, it is possible to obtain a better correction than the +0.07 found from the average of many samples, as previously described. This can be done by making tube measurements on a sample of the material at the time the sample is

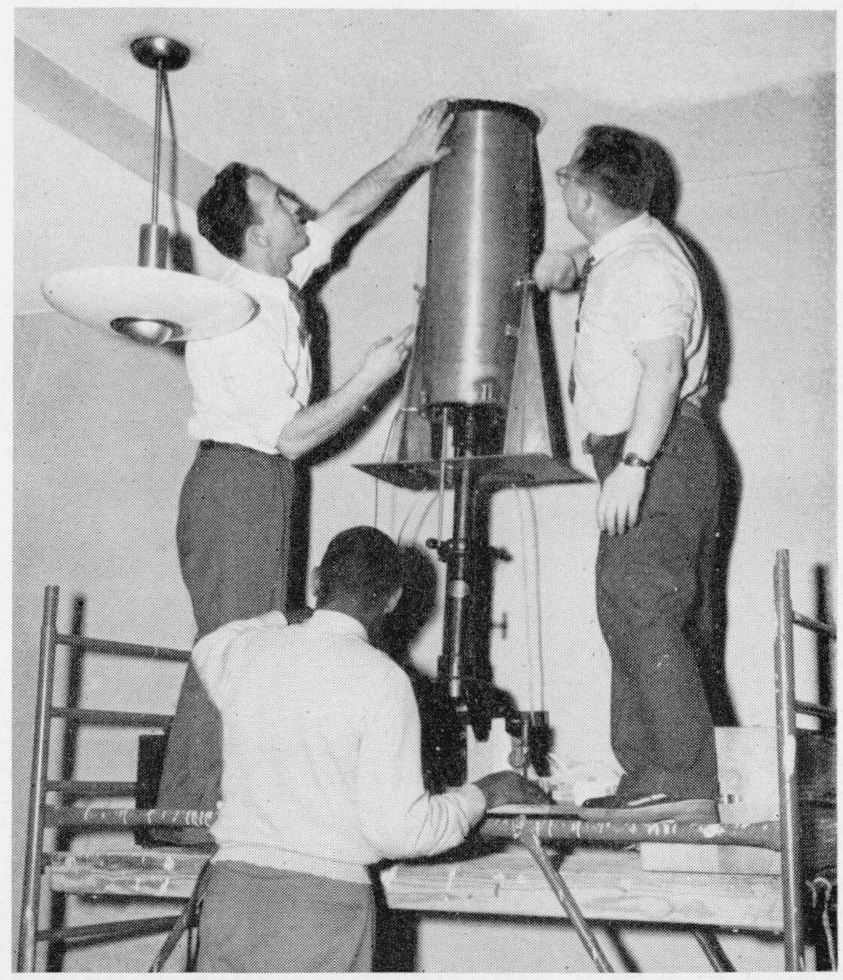

FIGURE 3. Field measurement of sound-absorpion coefficients being made by long-tube method. 
tested in the reverberation chamber. The coefficient at $512 \mathrm{c} / \mathrm{s}$ is also found by the tube method, averaging over many points on the same sample. The difference between the coefficients found by the reverberation-chamber method and by the tube method is taken as the correction to be applied in the field. This method gives a coefficient that will usually agree with the reverberation-room coefficient to within \pm 0.05 . Tube measurements taken on the same sample at different times have a maximum spread of only 0.03 .

The availability of a portable apparatus for nondestructive field testing makes it possible to correlate the appearance of a small area of acoustic plaster with its sound absorption. It is believed that the workmanship of the plasterer affects the sound absorption of the finished plaster to a large extent, but it is not known just how plaster should be applied for best results. The details of workmanship that are believed to affect sound absorption include the pressure of the trowel on the plaster, time of final troweling after application, consistency of mix, and the texture imparted to the surface. Different textures are obtained by the use of a stiff straw brush or a nail perforator, or both. Considerable difference of opinion exists concerning the importance of each of these factors and their proper manipulation. The development of the long-tube method makes possible objective evaluation of the various factors that are involved in the application of acoustic plasters.

The long-tube method makes it possible to measure the sound absorption of small areas, so that samples of many different examples of workmanship can be taken.

Other applications of the long-tube method have included absorption measurements made on the acoustic plaster of the ceilings of several construction projects for the Federal Government. The results of some of the tests are given in table 2 .

A recent example of the usefulness of the longtube method was the task of measuring the soundabsorption coefficients of two batches of commercial acoustic tiles. It was desired to select from each batch a smaller group of tiles nearly homogeneous in sound absorption for use in a series of round-robin laboratory tests. Figure 4 shows the percentage of the total number of $12-$ by 12 -in. tiles in each batch that had the indicated sound-absorption coefficient.

TABLE 2. Absorption measurements

\begin{tabular}{|c|c|c|c|c|}
\hline Location & . & $\begin{array}{l}\text { Number } \\
\text { of points } \\
\text { measured }\end{array}$ & $\begin{array}{c}\text { Range of } \alpha_{e}^{*} \\
\text { values }\end{array}$ & A verage $\alpha_{e}^{*}$ \\
\hline \multicolumn{5}{|c|}{ Building $\mathrm{A}$ at $512 \mathrm{c} / \mathrm{s}$} \\
\hline $\begin{array}{l}1 \\
2 \\
3 \\
4\end{array}$ & & $\begin{array}{r}33 \\
29 \\
7 \\
9\end{array}$ & $\begin{array}{l}0.24 \text { to } 0.63 \\
.25 \text { to } .45 \\
.32 \text { to } .41 \\
.15 \text { to } .47\end{array}$ & $\begin{array}{r}0.41 \\
.35 \\
.36 \\
.34\end{array}$ \\
\hline \multicolumn{5}{|c|}{ Building $\mathrm{B}$ at $512 \mathrm{c} / \mathrm{s}$} \\
\hline Fifth-floor ceiling... & & 42 & 0.28 to 0.62 & 0.49 \\
\hline
\end{tabular}

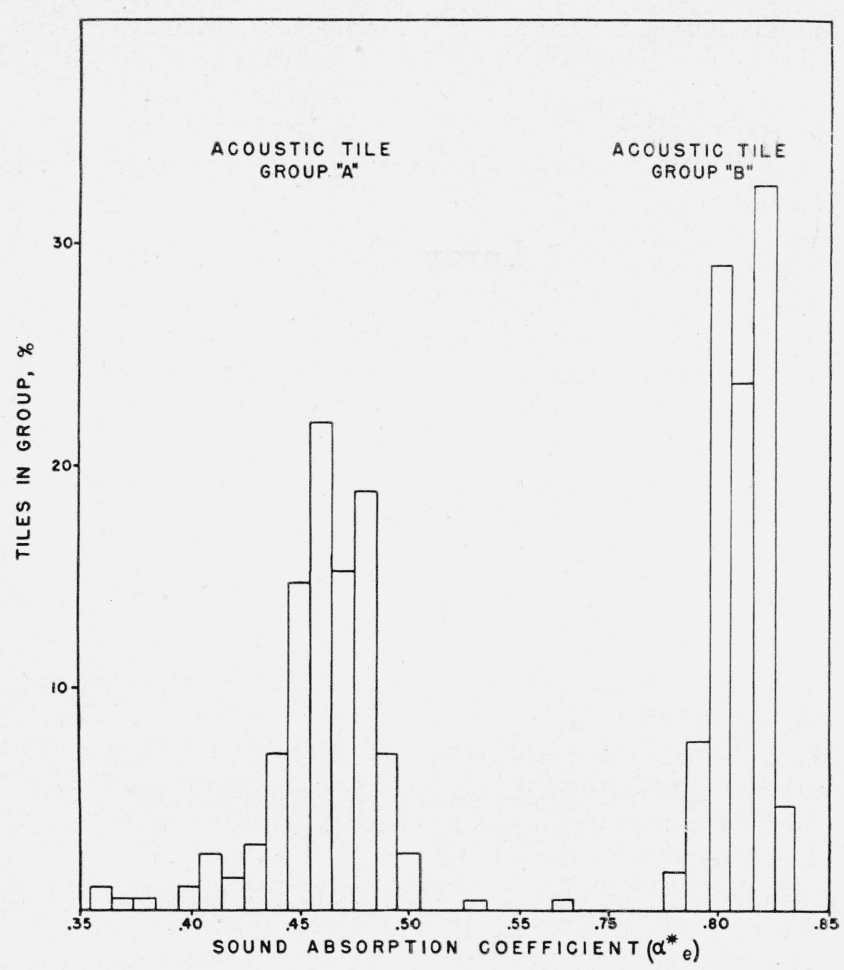

Figure 4. Comparison of distribution of sound-absorption coefficients in two different acoustic materials as measured by the long-tube method.

Sample A consisted of 108 12- by 12-in. tiles, and sample B consisted of 19512 by 12 -in. tiles.

\section{Conclusions}

The necessity of avoiding transverse modes sets an upper limit to the frequency at which a tube can be used. The lower limit of usable frequency is set by the requirement that the tube must not be too long for easy handling. These limits restrict the use of the tube described here to frequencies near $512 \mathrm{c} / \mathrm{s}$. However, other experiments indicate that if the formula used here to calculate the absorption coefficient is applied over the range of frequencies from 256 to $2,048 \mathrm{c} / \mathrm{s}$, the deviations between the tube method $\left(\alpha_{e}^{*}\right)$ and the reverberation-chamber method $\left(\alpha_{\text {rev }}\right)$ tend to be in opposite directions at different frequencies, so that the noise coefficient calculated from tube measurements agrees quite well with that calculated from reverberation-chamber measurements.

It would be possible to cover the frequency range from 256 to $2,048 \mathrm{c} / \mathrm{s}$ by making use of a separate tube of suitable size for each frequency. This has not been done because of the awkwardness of such an arrangement. However, it appears possible to replace the long tube with a short tube, which, in essence, is a tube so short as to constitute a cavity. Such a device would be readily portable, and should operate over an extended frequency range. A preliminary investigation of this method has been started.

Washington, April 16, 1952. 